\title{
Healthcare Costs for Metastatic Castration-Resistant Prostate Cancer Patients Treated with Abiraterone or Enzalutamide
}

\author{
Dikshyanta Rana ${ }^{1}$ (1) . Claudia Geue ${ }^{1}$ Kelly Baillie ${ }^{2}$. Jiafeng Pan ${ }^{3} \cdot$ Tanja Mueller $^{4} \cdot$ Jennifer Laskey $^{2}$. \\ Marion Bennie $^{4,5} \cdot$ Julie Clarke $^{2} \cdot$ Robert J. Jones $^{2,6} \cdot$ Ailsa Brown $^{7} \cdot$ Olivia Wu$^{1}$
}

Accepted: 29 September 2021 / Published online: 10 November 2021

(c) The Author(s) 2021

\begin{abstract}
Objective The aim was to assess the real-world healthcare resource use and direct medical costs for metastatic castrationresistant prostate cancer (mCRPC) patients treated with abiraterone or enzalutamide, in whom chemotherapy is not yet indicated (pre-chemotherapy) or who had previously received docetaxel-based chemotherapy (post-chemotherapy), before commencing these medicines.

Methods A retrospective cost analysis of mCRPC patients who commenced abiraterone or enzalutamide between 2012 and 2015 was conducted. Routinely collected datasets from the largest health board in Scotland and the UK, Greater Glasgow and Clyde, were linked. They contained information on patient demographics, diagnosis, outpatient consultations, hospital admissions, treatments (abiraterone and enzalutamide), and supportive medicines. Unit costs were obtained from the Scottish Health Service Costs, Personal Social Services Research Unit, and British National Formulary. Generalised linear model-based regression was used to estimate total mean direct costs, and two-part models were used to estimate separate cost components. All models were adjusted for propensity score and key variables. Sensitivity analysis was conducted to explore the impact of hypothetical patient access scheme discounts.

Results Estimated total mean direct medical costs of treating mCRPC patients were similar, albeit with wide and overlapping confidence intervals. Across both treatments, patients who received abiraterone or enzalutamide in a pre-chemotherapy setting incurred the highest total mean direct medical costs. However, post-chemotherapy patients were associated with higher outpatient clinic visits, inpatient hospital admissions, and supportive medicines. Regarding relative contribution to the total mean direct medical cost, the treatment costs were the main contributor, followed by inpatient admissions, outpatient clinic visits, and supportive medicines.

Conclusion The total mean direct medical costs were similar for abiraterone and enzalutamide patients. The costs were not driven by the choice of treatment regimen, but treatment setting (pre-chemotherapy or post-chemotherapy indications) and related healthcare resource utilisation. Future studies should focus on economic evaluations, such as cost-effectiveness analyses, using real-world data.
\end{abstract}

\section{Introduction}

Prostate cancer is the most common male cancer and the second most common cause of death in men in the UK [1]. An advanced form of prostate cancer is castration-resistant prostate cancer (CRPC) characterised by disease progression following bilateral orchidectomy or androgen deprivation therapy. CRPC accounts for $10-20 \%$ of all prostate

Dikshyanta Rana

dikshyanta.rana@glasgow.ac.uk

Extended author information available on the last page of the article cancer, of which $84 \%$ demonstrate radiographic findings of metastatic castration-resistant prostate cancer (mCRPC) [2]. mCRPC features poor prognosis, lack of curative treatments, and reduced survival compared to CRPC.

Recently, there have been advances in the treatment of mCRPC. Novel hormonal therapies like abiraterone and enzalutamide that target the androgen receptor are in use. The National Institute for Health and Care Excellence (NICE) and the Scottish Medicines Consortium (SMC) in the United Kingdom (UK) have deemed these medicines to be clinically effective and cost-effective for adults with mCRPC. Initially, these treatments were accepted for those with disease progression post docetaxel-based chemotherapy 


\section{Key Points for Decision Makers}

Our study demonstrates that the total mean direct medical costs for metastatic castration-resistant prostate cancer (mCRPC) patients treated with abiraterone or enzalutamide are similar.

The total mean direct medical costs in mCRPC patients were driven by treatment setting (pre-chemotherapy or post-chemotherapy indications) and related healthcare resource utilisation.

Resource utilisation and cost findings from this study can supplement future cost-effectiveness studies to make assumptions that reflect the real world.

$[3,4]$. Subsequently, both were accepted for use in mCRPC adults in whom chemotherapy is not yet indicated [5,6]. Abiraterone is taken orally at a dosage of $1000 \mathrm{mg}$ daily in combination with prednisolone, whereas enzalutamide is taken orally at $160 \mathrm{mg}$ daily [3-6]. Both medicines are taken until disease progression or drug toxicity.

Notably, reimbursement decisions made by the NICE and SMC are based on clinical evidence from key clinical trials and economic evidence from extrapolation of data obtained via multiple sources, including from experts. Thus, health technology assessments (HTAs) of new treatments, with sparse data, are subjected to uncertainties in estimates of clinical effectiveness and cost-effectiveness [3-6]. A recent systematic review [7] on cost-effectiveness analyses and cost analyses of mCRPC treatments confirms these uncertainties, including those attributed to misleading or missing cost information, and insufficient details provided by analyses in its consideration. Moreover, few costing studies [8-12] have to date utilised real-world data despite the expansion in prescription of abiraterone and enzalutamide to patients in the last decade. These studies utilised data obtained from claims and research databases such as the IBM MarketScan ${ }^{\circledR}$ from the providers' perspectives. Treatment duration and sequences, pharmacy costs, and healthcare resource utilisation in the form of outpatient consultations and hospital admissions were explored. However, the studies varied regarding treatment stratification, categorisation into prechemotherapy and post-chemotherapy, and inclusion of wide-ranging costs. Therefore, use of real-world data for this purpose has the potential to provide useful insight into the healthcare resource utilisation of mCRPC patients and also assist with making robust assumptions during costeffectiveness analyses.
Our study aims to assess the real-world healthcare resource use and direct medical costs for MCRPC patients treated with abiraterone or enzalutamide, in whom chemotherapy is not yet indicated or who had previously received docetaxel-based chemotherapy, before commencing these treatments. The study will inform clinicians on how mCRPC patients treated with abiraterone and enzalutamide in the UK engage within routine care settings in terms of healthcare resource utilisation as represented by costs.

\section{Methods}

This retrospective cohort cost analysis was conducted as a part of the Cancer Medicines Outcomes Programme (CMOP) funded within the Scottish government's "Beating Cancer: Ambition and Action" Cancer Plan [13]. CMOP aims to facilitate the use of electronic record linkage of routinely collected data in Scotland to allow the outcomes of cancer medicines in real-life clinical practice to be better understood. Our analysis complements the recently published clinical paper that demonstrated treatment outcomes and trial eligibility in real-world mCRPC patients in Scotland [14].

Several routinely collected datasets were accessed to gather data on all mCRPC patients who commenced abiraterone or enzalutamide between February 2012 and December 2015 in Greater Glasgow and Greater Clyde (GG and C). GG and C is the largest health board in Scotland and the UK, which provides healthcare for 1.2 million people. The study's sample size is determined by the chosen dates which correspond to the approval of both medicines in Scotland and data availability for appropriate follow-up [14]. mCRPC patients prescribed abiraterone or enzalutamide were identified via the Chemotherapy Electronic Prescribing and Administration System (CEPAS). CEPAS is a comprehensive source of data of all systemic anti-cancer therapy (SACT) prescriptions within the National Health Service (NHS) Scotland [15]. Patients who commenced abiraterone or enzalutamide during the recruitment period or were part of a clinical trial, except when the drug was used within its product label, were excluded from the study. Similarly, patients who were prescribed either of the treatments but did not commence it were excluded. The earliest index date and the last date of data collection for patients who were still alive was February 2012 and 28 February 2017, respectively. The date of the final dose of the medicines or date of death (if patients died while on treatment) was entered as the treatment end date. Patients were followed up until the treatment end date or the censor date, whichever occurred first. All patients commenced the treatments in two settings (indications): the pre-chemotherapy setting included those in whom chemotherapy was not yet indicated, also called as 
chemotherapy naïve, while the post-chemotherapy setting included patients who had previously received docetaxelbased chemotherapy.

The datasets were linked via a unique patient identifier, i.e. the Community Health Index (CHI) number, which is used throughout the NHS to identify individual patients. Further detail on this is available in our clinical paper [14]. The researcher received fully anonymised data for this analysis. A variety of datasets containing information on patient demographics, details of diagnosis, treatments, outpatient appointments, and hospital admissions were available. This cost analysis utilised CEPAS, Scottish Morbidity Records (SMR), and National Records of Scotland (NRS) (Table 1).

CEPAS provided information on chemotherapy medicines' prescription, including SACTs and supportive medicines (steroids, anti-emetics). Supportive medicines do not include medicines dispensed in the community. Outpatient clinic visit information was obtained from the SMR-00 records [16], including information on any new and followup appointments. Inpatient hospital admission information was obtained from SMR-01 [17], which records all acute general admissions as inpatient or day cases. Inpatient stays indicate a hospital stay overnight, while day cases indicate planned attendance of a specialty clinic. Unlike outpatient records, day cases include patients who do not necessarily stay overnight, but require a hospital bed. A record is generated on completion of an episode as determined by the admission date and discharge date. NRS [18], register of all life events, was used to collect mortality information. It records each death in Scotland, along with relevant International Classification of Diseases 10th Revision (ICD-10) codes.

The analysis was conducted from the perspective of NHS Scotland. Resource use data consisted of items such as the number of outpatient clinic visits, length of stay for inpatient admissions, and the frequency, dose, and duration of treatment (including main medicines abiraterone administered with prednisolone or enzalutamide) and supportive medicines. Unit costs were assigned using the Scottish Health Service Costs ('cost book') [19], Personal Social Services Research Unit (PPSRU) [20], and the British National Formulary (BNF) [21]. Some assumptions and adjustments were made during this process:

1. Unit costs for outpatient clinic visit and inpatient hospital admission-related resource items were derived from health board-level average costs available in the cost book, according to the specialty, as the study population consisted of GG and C patients only.

2. Pharmacy cost was deducted from the cost book's direct cost per case. This was necessary as the average direct cost per case is calculated and published by including all medical and dental costs, costs for nursing, pharmacy and allied health professionals, and, theatre and laboratory costs [19]. This deduction avoids double counting of prescription costs available from the CEPAS.

3. An added cost of referral was attached to all new outpatient appointments. It captured the cost of new patients who were referred to an outpatient clinic for further tests or treatments by healthcare professionals such as general practitioners (GPs).

4. Patients were assigned unit costs according to their prescribed dose. Standard BNF doses were assumed where data on dosages were missing.

5. The duration of medicine prescription was rounded to the nearest 28 days as patients would have received each prescription for a minimum of this period regardless of whether they stopped early. This was counted as a cycle of medicine.

6. No administration cost was assumed to be incurred, as most of the medicines were administered orally.

Per diem costing was used [22]. All costs were expressed in UK pounds sterling (f) for the price year $2018 / 2019$.

Table 1 Routinely collected datasets used in the cost analysis

\begin{tabular}{ll}
\hline Source & Example of data \\
\hline CEPAS & $\begin{array}{c}\text { Prescription of chemotherapy treatments (abiraterone, enzalutamide, and supportive medicines) and other information such as } \\
\text { appointment date, intention (palliative or curative)/type of treatment, regime, cycle, dose, duration, and frequency of the drugs is } \\
\text { also recorded } \\
\text { Outpatient clinic visits, including information on new and follow-up appointments, and additional information on specialty, the } \\
\text { professionals who led the clinic, and the date of attendance }\end{array}$ \\
SMR 00* $01^{*}$ & $\begin{array}{c}\text { All acute general admissions in the form of inpatient and day cases and additional information on specialty, date of admission/ } \\
\text { discharge/transfer, type of admissions (elective or non-elective)/discharge (regular, self-discharge, or death), and reason for } \\
\text { admission (ICD-10) }\end{array}$ \\
Date of death and cause of death
\end{tabular}

CEPAS Chemotherapy Electronic Prescribing and Administration System, ICD-10 International Classification of Diseases 10th Revision, NRS National Records of Scotland, SMR Scottish Morbidity Records

*Also covers information related to demographics, socio-economic status, health board area, and hospital 
Further information on resource use items, unit costs, and their sources are presented as supplementary information (see the electronic supplementary material, online resource Table S1).

Total cost per patient was calculated by assigning unit costs to each patient's resource use items throughout the study period. Cost per outpatient clinic visit was determined by specialty and professional who led the clinic. Cost per inpatient hospital admission depended on the specialty and length of stay in the hospital; they were multiplied to calculate total cost per episode. Length of stay was calculated as the difference in days between admission date and discharge date, including these days. The total inpatient hospital admission cost per patient was calculated by summing up the total cost for each episode. For medicines, the price per unit was obtained by dividing the price per pack by pack size, adjusted for doses. The unit cost of medicine was multiplied with the prescription's frequency and duration to obtain the cost of medicine per day and total cost per prescription, respectively. The total medicine cost per patient was calculated by summing up the total cost per prescription. The total direct medical cost per mCRPC patient therefore included outpatient clinic visits cost and cost of referral, inpatient hospital admission cost, cost of treatment (main medicines abiraterone plus prednisolone or enzalutamide), and cost of supportive medicines.

For the analysis, patients were categorised into four groups based on treatment regimens (abiraterone or enzalutamide) and settings (indications) of the medicine (pre- and post-chemotherapy). Descriptive statistics were obtained for comparing the baseline characteristics. To address potential bias due to observed confounding, we estimated propensity scores using a logit model. Conditional probabilities were predicted, and weights generated as the inverse of the observed probability of receiving a particular treatment. Balance between covariates after weighting was assessed. Covariate selection for weight estimation was determined by covariate measurement at baseline and its clinical relevance. Key covariates included type of chemotherapy setting, age, Charlson Comorbidity Index (CCI), and Scottish Index of Multiple Deprivation (SIMD). In line with the clinical study, age was categorised as less than and more than 75 years. The CCI categorises comorbidities according to ICD-codes and assigns associated weights from one to six [23, 24]. The CCI score was re-categorised as 0,1 , and 2 or more; 0 indicates no comorbidities, while a higher CCI score indicates severe comorbidities. SIMD is a measure of deprivation considering seven criteria including income and health [25]. It is measured in quintiles, ranging from 1 (most deprived quintile) to 5 (least deprived quintile). Non-normal distribution of costs and presence of excess zeroes were addressed using two-part models [26]. The first part estimated the probability of observing positive (non-zero) costs with a logit model for those cost components with zero cost observations. In the second modelling part, costs were estimated conditional on positive healthcare utilisation using generalised linear models (GLMs) with a Gamma distribution and a log link. The appropriate family and link were selected based on the modified Park test and other tests [27]. Using the doublerobust method for propensity score adjustment, all models were adjusted using inverse probability weighting as well as all key covariates.

A sensitivity analysis was performed to explore the impact of a patient access scheme (PAS) on the cost of treatments. The PAS is a specified maximum price or a discount that tracks any changes to the UK medicine list price and enables the NHS to provide better access to innovative medicines [28]. As the PAS discounts are confidential, we applied hypothetical discounts of $25 \%$ and $50 \%$ on abiraterone and enzalutamide prices, which were based on BNF unit costs. Though abiraterone and prednisolone were grouped together, the discount was applied only to the former. Note that hypothetical PAS discounts do not imply anything about the range or level of discounts applicable in practice. All analyses were carried out in Stata version 15.0 (College Station, TX, US).

\section{Results}

Overall, $261 \mathrm{mCRPC}$ patients commenced abiraterone ( $n$ $=145$ ) or enzalutamide $(n=116)$ (Table 2$)$. More patients had previously received a docetaxel-based chemotherapy before commencing either of the treatment regimens (postchemotherapy setting, abiraterone $n=82$; enzalutamide $n=$ 74). Pre-chemotherapy patients were older on average. Most patients had no comorbidities (CCI score of 0 ). More severe comorbidities (CCI score of 2 or more) were observed for pre-chemotherapy patients compared to post-chemotherapy patients. The SIMD shows that the majority of pre-chemotherapy patients lived in the most deprived areas of Scotland, whilst the majority of post-chemotherapy patients lived in the least deprived areas of Scotland. Pre-chemotherapy patients also received greater median cycles of treatment (number of times a patient gets prescribed abiraterone or enzalutamide) compared to post-chemotherapy patients. They were also on treatment for longer as shown by the time to treatment discontinuation. Thus, the overall observed follow-up was longer for pre-chemotherapy patients. During the follow-up, patients in the abiraterone and enzalutamide post-chemotherapy setting attended outpatient clinics more frequently than those in pre-chemotherapy settings. The number of inpatient hospital admissions and length of stay were similar across all groups. Post-chemotherapy patients had a slightly longer length of stay. A typical patient treatment pathway featuring healthcare resource utilisation is 
Table 2 Patient characteristics, treatment details, and healthcare resource use

\begin{tabular}{|c|c|c|c|c|}
\hline & \multicolumn{2}{|l|}{ Abiraterone $(n=145)$} & \multicolumn{2}{|c|}{ Enzalutamide $(n=116)$} \\
\hline & Pre-chemo $(n=63)$ & Post-chemo $(n=82)$ & Pre-chemo $(n=42)$ & Post-chemo $(n=74)$ \\
\hline \multicolumn{5}{|l|}{ Patient characteristics } \\
\hline Age, mean (SD), years & $74.9(8.1)$ & $71.4(7.4)$ & $76.9(6.0)$ & $71.9(8.5)$ \\
\hline$\leq 75, n(\%)$ & $34(54.0)$ & $56(68.3)$ & $16(38.1)$ & $48(64.9)$ \\
\hline $75+, n(\%)$ & $29(46.0)$ & $26(31.7)$ & $26(61.9)$ & $26(35.1)$ \\
\hline \multicolumn{5}{|l|}{ CCI score, $n(\%)$} \\
\hline 0 & $37(58.7)$ & $51(62.2)$ & $24(57.1)$ & $53(71.6)$ \\
\hline 1 & $12(19.0)$ & $15(18.3)$ & $8(19.0)$ & $8(10.8)$ \\
\hline 2 or more & $14(22.2)$ & $16(19.5)$ & $10(23.8)$ & 13 (17.6) \\
\hline \multicolumn{5}{|l|}{ SIMD quintiles, $n(\%)$} \\
\hline 1 (most deprived) & $19(30.2)$ & $22(26.8)$ & $15(35.7)$ & $16(21.6)$ \\
\hline 2 & $10(15.9)$ & $10(12.2)$ & $5(11.9)$ & $13(17.6)$ \\
\hline 3 & $9(14.3)$ & $10(12.2)$ & $7(16.7)$ & $10(13.5)$ \\
\hline 4 & $9(14.3)$ & $12(14.6)$ & $8(19.0)$ & $9(12.2)$ \\
\hline 5 (Least deprived) & $16(25.4)$ & $27(32.9)$ & $7(16.7)$ & $23(31.1)$ \\
\hline Unknown & & $1(1.2)$ & & $3(4.1)$ \\
\hline \multicolumn{5}{|l|}{ Treatment details } \\
\hline Number of cycles: mean (SD); median (IQR) & $\begin{array}{l}10.8(6.7) \\
11(5-16)\end{array}$ & $\begin{array}{l}10.9(9.4) \\
8.5(4-14)\end{array}$ & $\begin{array}{l}12.2(9.7) \\
11(4-18)\end{array}$ & $\begin{array}{l}9.8(8.6) \\
7(3-15)\end{array}$ \\
\hline $\begin{array}{l}\text { Time to treatment discontinuation in months: mean } \\
\text { (SD); median (IQR) }\end{array}$ & $\begin{array}{l}10.1(7.1) \\
10(4.5-14.7)\end{array}$ & $\begin{array}{l}9.3(9) \\
6.7(2.7-12.9)\end{array}$ & $\begin{array}{l}10.7(9.3) \\
10(3.1-16.5)\end{array}$ & $\begin{array}{l}8.6(8.4) \\
5.7(2.2-14.5)\end{array}$ \\
\hline $\begin{array}{l}\text { Observed follow-up months: mean (SD); median } \\
\text { (IQR) }\end{array}$ & $\begin{array}{l}14.8(7.6) \\
15.0(11.3-16.4)\end{array}$ & $\begin{array}{l}13.0(10.5) \\
10.7(4.5-18.3)\end{array}$ & $\begin{array}{l}14.1(9.4) \\
15.6(4.7-20.4)\end{array}$ & $\begin{array}{l}14.6(10.6) \\
12.6(5.3-22.6)\end{array}$ \\
\hline \multicolumn{5}{|l|}{ Healthcare resource use } \\
\hline $\begin{array}{l}\text { Number of outpatient clinic visits: mean (SD); median } \\
\text { (IQR) }\end{array}$ & $\begin{array}{l}11.9(9.4) \\
9(5-17)\end{array}$ & $\begin{array}{l}14.7(10.2) \\
13(7-21)\end{array}$ & $\begin{array}{l}8.7(6.7) \\
7(4-12)\end{array}$ & $\begin{array}{l}11.5(9.0) \\
10(5-16)\end{array}$ \\
\hline \multicolumn{5}{|l|}{ Most frequent specialties in outpatient clinic visits, $n(\%)$} \\
\hline 1.Clinical oncology & $359(47.8)$ & $750(63.7)$ & $125(36.0)$ & $484(57.8)$ \\
\hline 2.Medical oncology & $255(33.9)$ & $295(25.1)$ & $132(38.0)$ & $264(31.5)$ \\
\hline 3.Ophthalmology & $12(1.6)$ & $25(2.1)$ & $13(3.8)$ & $16(1.9)$ \\
\hline $\begin{array}{l}\text { Number of inpatient hospital admissions: mean (SD); } \\
\text { median (IQR) }\end{array}$ & $\begin{array}{l}6.5(5.2) \\
5(3-9)\end{array}$ & $\begin{array}{l}6.3(5.5) \\
5(3-7)\end{array}$ & $\begin{array}{l}5.1(4.7) \\
4(2-5)\end{array}$ & $\begin{array}{l}6.2(4.8) \\
5(3-8)\end{array}$ \\
\hline Length of stay: mean (SD); median (IQR) & $\begin{array}{l}5.2(8.6) \\
2(1-5)\end{array}$ & $\begin{array}{l}6.9(9.7) \\
3(1-8)\end{array}$ & $\begin{array}{l}5.9(9.0) \\
2(1-5)\end{array}$ & $\begin{array}{l}6.3(11.0) \\
2(1-7)\end{array}$ \\
\hline \multicolumn{5}{|c|}{ Most frequent specialties in inpatient hospital admissions, $n(\%)$} \\
\hline 1.General medicine & $88(27.9)$ & $144(31.1)$ & $51(30.2)$ & $150(34.3)$ \\
\hline 2.Clinical oncology & $47(14.9)$ & $60(13.0)$ & $9(5.3)$ & $84(9.2)$ \\
\hline 3.Urology & $30(9.5)$ & $76(16.4)$ & $22(13.0)$ & $21(4.8)$ \\
\hline
\end{tabular}

CCI Charlson Comorbidity Index, IQR interquartile range, SIMD Scottish Index of Multiple Deprivation

available as supplementary information (see the electronic supplementary material, online resource Figure S1).

The estimated total mean direct medical costs of mCRPC patients treated with abiraterone and enzalutamide were very similar (Table 3). The total mean cost for abiraterone and enzalutamide patients was $£ 53,808$ (95\% confidence interval [CI] 47,377-60,239) and £55,652 (95\% CI 48,019-63,285), respectively. These costs are estimated for the entire period between index and censor date and not annualised. GLM regression results for total mean direct medical cost (see the electronic supplementary material, online resource Table S2) showed that post-chemotherapy patients had lower total mean costs compared to pre-chemotherapy patients. Also, patients older than 75 years had lower costs than their younger counterparts. Both associations were not statistically significant. Patients with a higher CCI score (1 and 2 or more) incurred lower total mean costs than those with a score of 0 . Only the association between a CCI score of 2 or more and total mean costs was statistically significant $(p<0.05)$. Patients in an SIMD quintile greater than 1 had 
higher total mean costs; however, this association was only statistically significant for patients living in SIMD quintile $4(p<0.05)$. The GLM regression results for total cost and individual cost components based on treatment regimen are available in the supplementary online resource (see the electronic supplementary material, online resource Table S3 and S4).

When the total mean direct medical cost is categorised according to treatment regimens and type of chemotherapy settings (Table 3), the cost remains very similar. Patients who had abiraterone or enzalutamide treatments pre-chemotherapy had a slightly higher cost. However, after treatment discontinuation, post-chemotherapy patients incurred almost double the total direct medical cost. Regarding relative contribution to the total mean direct medical costs, the treatment costs were found to be the main contributor, followed by inpatient hospital admissions, outpatient clinic visits, and supportive medicines (Figure 1). The total mean cost of treatment was greater for patients belonging to prechemotherapy settings. Conversely, the total mean costs of outpatient clinic visits, inpatient hospital admissions, and supportive medicines for post-chemotherapy patients in both treatment groups were higher compared to those for prechemotherapy patients.
Our sensitivity analysis (Table 4), where PAS discounts were applied to abiraterone and enzalutamide prices, revealed similar results as the main analysis, with the difference in costs between the four groups being minimal. With a $25 \%$ discount, post-chemotherapy abiraterone patients and pre-chemotherapy enzalutamide patients incurred higher costs compared to their counterparts. With a 50\% discount, both post-chemotherapy abiraterone and enzalutamide patients had higher total costs (Fig. 2). All cost estimates remained above $£ 30,000$ per patient.

\section{Discussion}

Our evidence showed that the estimated total mean direct medical costs of treating mCRPC patients, in whom chemotherapy is not yet indicated (pre-chemotherapy) or who had previously received docetaxel-based chemotherapy (postchemotherapy), with abiraterone or enzalutamide were similar, albeit with wide and overlapping CIs. The costs were not driven by choice of treatment regimens, but treatment settings (indications) and related healthcare resource utilisation.

The breakdown of cost into components demonstrated that the treatment costs itself contributed most to the total

Table 3 Total mean direct medical costs (£) of mCRPC patients treated with abiraterone or enzalutamide

\begin{tabular}{|c|c|c|c|c|}
\hline \multirow{3}{*}{$\begin{array}{l}\text { Total mean direct medical cost } \mathrm{t}^{\dagger} \text { of } \\
\text { mCRPC patients, according to treat- } \\
\text { ment regimens }\end{array}$} & \multicolumn{2}{|c|}{ Abiraterone cost $(\mathfrak{f})(95 \% \mathrm{CI})$} & \multicolumn{2}{|c|}{ Enzalutamide cost $(\mathfrak{f})(95 \% \mathrm{CI})$} \\
\hline & $\begin{array}{l}53,808 \\
(47,377-60,239)\end{array}$ & & $\begin{array}{l}55,652 \\
(48,019-63,285)\end{array}$ & \\
\hline & $\begin{array}{l}\text { Pre-chemo } \\
\text { Mean (95\% CI) }\end{array}$ & $\begin{array}{l}\text { Post-chemo } \\
\text { Mean (95\% CI) }\end{array}$ & $\begin{array}{l}\text { Pre-chemo } \\
\text { Mean }(95 \% \text { CI) }\end{array}$ & $\begin{array}{l}\text { Post-chemo } \\
\text { Mean }(95 \% \text { CI) }\end{array}$ \\
\hline $\begin{array}{l}\text { Total mean direct medical cost } \mathrm{t}^{\dagger} \text { of } \\
\text { mCRPC patients, according to treat- } \\
\text { ment regimens and types }\end{array}$ & $54,815(44,963-64,667)$ & $53,394(44,792-61,995)$ & $56,907(43,617-70,197)$ & $54,919(45,854-63,984)$ \\
\hline $\begin{array}{l}\text { Total mean direct medical cost }{ }^{\dagger} \text { of } \\
\text { mCRPC patient, after treatment } \\
\text { discontinuation }\end{array}$ & $5986(3217-8756)$ & $10,548(6985-14,111)$ & $5576(2938-8214)$ & $10,330(7090-13,569)$ \\
\hline \multicolumn{5}{|c|}{ Total mean cost of healthcare resource use components ${ }^{\S}$} \\
\hline Outpatient clinic visits & $1419(1128-1709)$ & $1603(1332-1874)$ & $977(717-1237)$ & $1252(1018-1486)$ \\
\hline Inpatient hospital admissions & $13,176(8218-18,133)$ & $18,071(13,626-22,517)$ & $12,170(7513-16,827)$ & $17,557(13,830-21,284)$ \\
\hline $\begin{array}{l}\text { Treatment (main medicines abirater- } \\
\text { one }+ \text { prednisolone or enzaluta- } \\
\text { mide) }\end{array}$ & $40,606(32,973-48,238)$ & $33,402(27,058-39,747)$ & $44,080(30,483-57,677)$ & $36,010(27,634-44,387)$ \\
\hline Supportive medicines ${ }^{\ddagger}$ & $54(15-93)$ & $216(141-291)$ & $46(27$ to 119$)$ & $64(15-113)$ \\
\hline
\end{tabular}

$C I$ confidence interval, $m C R P C$ metastatic castration-resistant prostate cancer

All mean costs represent computed predictions from generalised linear models and not arithmetic means. All costs were in pounds sterling, reflecting 2018 prices

${ }^{\dagger}$ Total mean direct medical cost per patient included the following: outpatient clinic visits cost and cost of referral; inpatient hospital admissions costs; treatment cost (main medicines abiraterone plus prednisolone or enzalutamide); and cost of supportive medicines. All abiraterone patients were prescribed prednisolone. Unit cost of prednisolone is very minimal compared to abiraterone

${ }^{\S}$ Cost components apart from treatment costs obtained from two-part model

${ }^{\ddagger}$ Supportive medicines include any other medicine prescribed to the patients along with the main medicines 


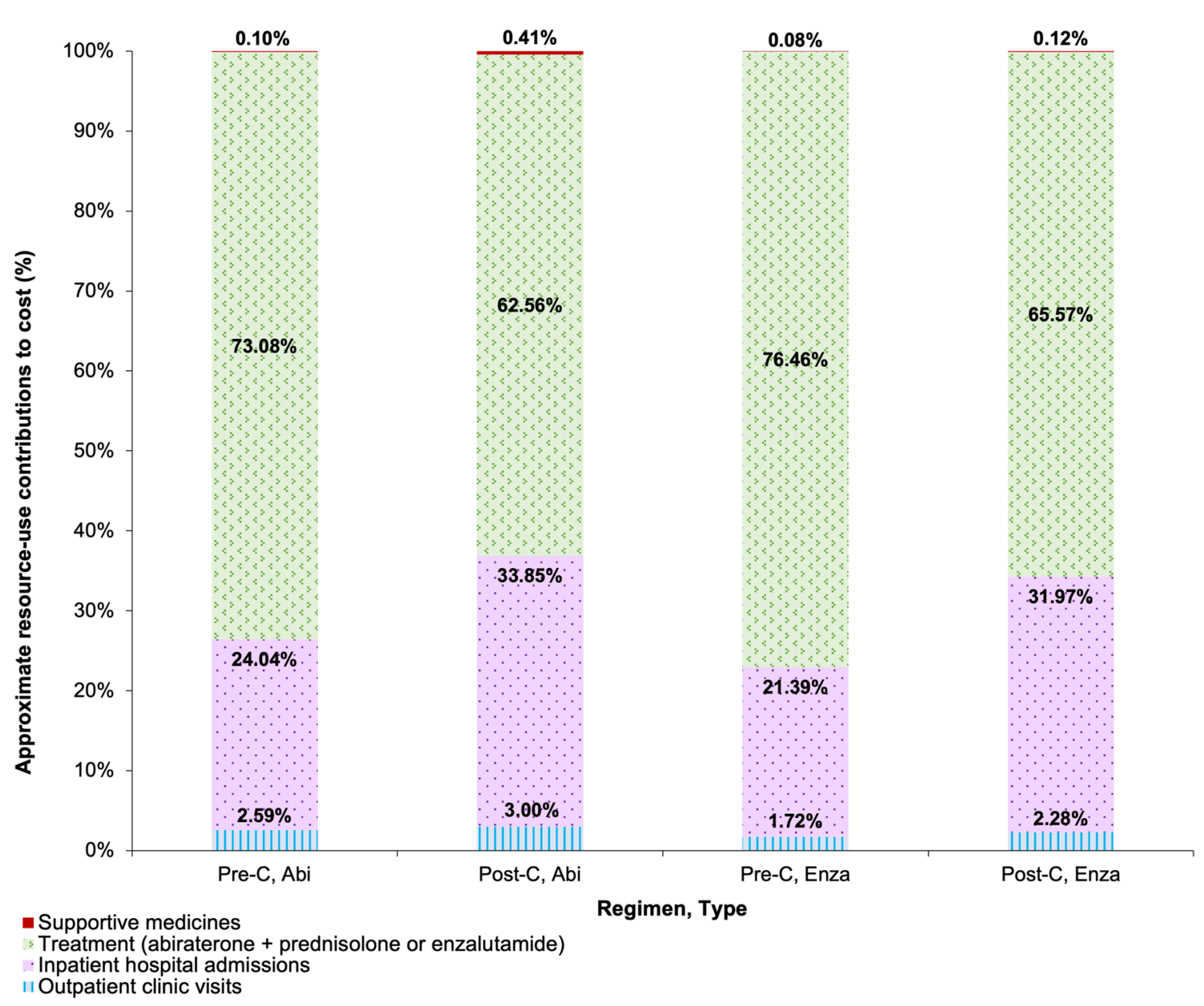

Fig. 1 Contribution of resource use components to the total mean direct medical costs. Abi abiraterone, Enza enzalutamide, Post-C post-chemotherapy, Pre-C pre-chemotherapy

Table 4 Total mean direct medical costs (£) after application of $25 \%$ and $50 \%$ hypothetical discounts on treatment costs to imitate patient access schemes

\begin{tabular}{|c|c|c|c|c|}
\hline Discount & Abiraterone $(£)(95 \% \mathrm{CI})$ & & Enzalutamide (f) $(95 \% \mathrm{CI})$ & \\
\hline $25 \%$ & $44,758(39,373-50,144)$ & & $45,972(39,916-52,027)$ & \\
\hline \multirow[t]{2}{*}{$50 \%$} & $35,730(31,278-40,182)$ & & $36,276(31,677-40,874)$ & \\
\hline & Pre-chemo & Post-chemo & Pre-chemo & Post-chemo \\
\hline $25 \%$ & $44,745(36,454-53,036)$ & $45,040(37,781-52,300)$ & $45,962(35,607-56,318)$ & $45,904(38,664-53,144)$ \\
\hline $50 \%$ & $34,695(27,813-41,577)$ & $36,680(30,649-42,712)$ & $35,039(27,354-42,723)$ & $36,883(31,307-42,458)$ \\
\hline
\end{tabular}

CI confidence interval

All mean costs represent computed predictions from generalised linear models and not arithmetic means

mean direct medical costs, followed by inpatient hospital admissions, outpatient clinic visits and supportive medicines. In our study, pre-chemotherapy patients had higher treatment costs as these patients had a greater number of treatment cycles and a longer time to treatment discontinuation, irrespective of their treatment regimen. They also had a longer observed follow-up. However, these patients had relatively lower resource utilisation and costs of other healthcare components (outpatient clinic visits, inpatient hospital admissions, supportive medicines). Instead, we observed that post-chemotherapy patients in both treatment regimens had higher resource utilisation. Relatedly, the findings from 


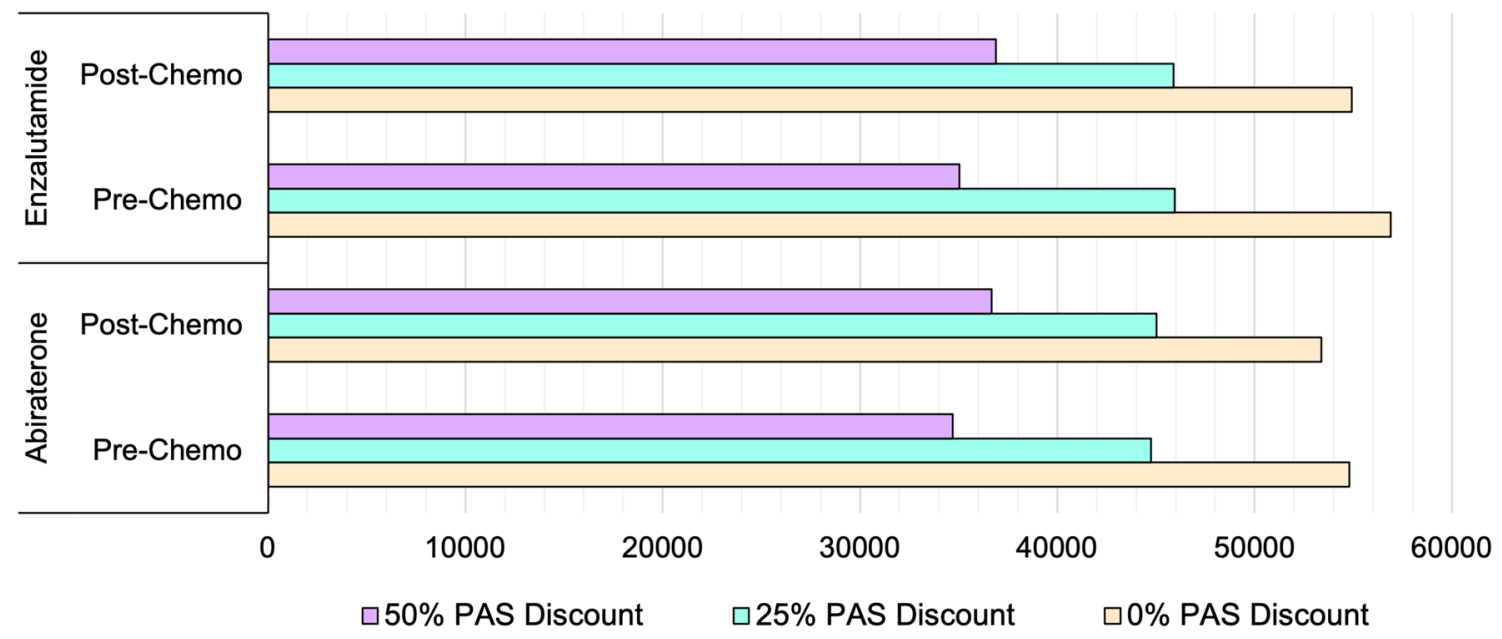

Fig. 2 Comparison of total cost (f) according to treatment regimens and medicine settings after application of $25 \%$ and $50 \%$ hypothetical discounts on treatment costs to imitate patient access schemes (PAS)

our clinical paper [14] showed that post-chemotherapy patients had lower median overall survival [abiraterone 10.8 months (95\% CI 8.6-15.1); enzalutamide 12.6 months (95\% CI 10.5-18.2)] than pre-chemotherapy patients [abiraterone 20.9 months (95\% CI 14.9-29); enzalutamide 16 months (95\% CI 9.8 to 'not reported')]. Though these patients had shorter durations on either abiraterone or enzalutamide, as reflected by lower treatment costs, they could have sought medical help frequently due to their deteriorating health. Indeed, they had a consistently greater number of outpatient clinic visits and inpatient hospital admissions with slightly longer length of stay throughout, and also utilised more supportive medicines. Moreover, post-chemotherapy patients continued to incur almost double the total mean direct medical costs compared to pre-chemotherapy patients after treatment discontinuation despite there being a short time until censor date. This increased accrual of costs could also be attributed to potential excess resource use during hospital admission at the end of life.

Overall, pre-chemotherapy patients still incurred the highest total mean direct medical costs, albeit very similar between the two treatment regimens. Here, the costs associated with other resource use components did not offset the difference in treatment cost-thus, revealing the expensive nature of both treatments. Therefore, this explains similar overall total costs between both treatment regimens, for which our clinical paper [14] also estimated similar median overall survival [abiraterone patients 14.6 months (95\% CI 12.3-17.0); enzalutamide patients 14.0 months (95\% CI 11.5-18.2)].

Sensitivity analysis validates our findings as total mean direct medical costs between the groups were very similar. The costs were still similar even when it flipped in favour of other settings in some scenarios. The analysis further establishes that choice of mCRPC treatment regimen does not sufficiently explain the differences in total mean direct medical cost between the groups. It showcases the expensive nature of abiraterone and enzalutamide even when PAS were applied.

There were a handful of studies based on real-world data with which we could compare our results. The treatment stratification in these studies differed: one focused only on abiraterone patients [9], none focused on enzalutamide patients exclusively, and four focused on both treatments [8, 10-12]. The studies also varied in their focus of settings: three included only pre-chemotherapy setting [10-12] and two included both pre- and post-chemotherapy settings [8, 9]. In most studies including both treatments, patients were frequently prescribed abiraterone [8,10-12]. This pattern was observed in our study as well. The majority of studies conclude that the total healthcare costs of abiraterone and enzalutamide patients did not differ much [8, 10, 12]. One claims that total cost was lower for enzalutamide-treated patients than for abiraterone-treated patients [11]. Regarding the drivers of total costs of treating mCRPC patients, available literature features result similar to our cost components. Treatment costs (drug cost) itself were a major driver of total costs $[8,9]$. Even though abiraterone patients were prescribed prednisone concurrently, pharmacy costs were lower for them compared to enzalutamide patients $[8,10]$. This was true in case of our results. Contrastingly, a study observed enzalutamide patients to have lower medical/pharmacy costs [11]. Treatment duration was longer for enzalutamide patients [10], whilst in our study, we had similar treatment durations between the groups. We also observed that the majority of our patients utilised supportive medicines $[8$, 10]. Additionally, the studies showed enzalutamide patients to have lower costs associated with inpatient admissions, 
including emergency department visits, and outpatient visits compared with those for abiraterone patients [10,11]. Our cost components show similar results. Any comparison between the studies must be interpreted cautiously as the studies' populations comprised different treatment stratifications, settings (indications), and distinct characteristics. Moreover, some costs were observed on a monthly/yearly basis and only included pharmacy costs.

This is the first study using real-world evidence that distinctly includes both pre- and post-chemotherapy patients in the UK and captures their healthcare resource utilisations during the treatment period and after treatment discontinuation. It has demonstrated the value of routine linked health data in evaluating new treatments by capturing a broader population than clinical trials and presenting information that goes beyond the end of the treatment period. Our results highlight the expensive nature of both abiraterone and enzalutamide through the main results and hypothetical PAS discount to shed light on the burden of mCRPC on the provider, i.e. the NHS. It should be noted that abiraterone and enzalutamide are recommended by the NICE in accordance with the commercial access arrangement.

There are some limitations to our analysis. As mentioned, our small sample size can be attributed to the timing of abiraterone and enzalutamide approval in Scotland and data availability for appropriate follow-up [14]. Nevertheless, we have included all patients who started either of the treatments between February 2012 and December 2015 in $\mathrm{GG}$ and $\mathrm{C}$ area. There was an imbalance in the number of patients in all four groups, due to earlier HTA acceptance of abiraterone, particularly for post-chemotherapy patients [3-6], which led to more mCRPC patients being prescribed abiraterone. Data related to key clinical variables such as Gleason score may have added more granularity to our analysis, but was not available for all patients. Per diem costing assumes that the cost for the first day in hospital is equal to any subsequent days [22]. However, this is likely to have a negligible impact on our results. Low-cost medicines prescribed in the community were not included and would be unlikely to change our results. The analysis maybe subject to some residual and unobserved confounding. GLM results feature wide CIs for the covariates, which indicates that the sample size was too small. The estimated total costs also presented very wide and overlapping CIs.

In conclusion, direct medical costs for mCRPC patients treated with abiraterone or enzalutamide are similar. Our study provides an understanding of $\mathrm{MCRPC}$ in routine care and has the potential to supplement future resource allocation decisions aimed at improving efficiency. Future studies should focus on economic evaluations, such as cost-effectiveness analyses, using real-world evidence. Studies that further explore factors such as drug wastage, adherence, toxicity, adverse events, and additional factors that can contribute to better patient management are warranted.

Supplementary Information The online version contains supplementary material available at https://doi.org/10.1007/s41669-021-00307-1.

Acknowledgements We thank the Scottish Government and the wider Cancer Medicines Outcomes Programme (CMOP) group. The availability of this rich dataset is possible because of the routine information collected by the NHS.

\section{Declarations}

Funding This work was supported by the Scottish Government. The funder had no role in the study design, data collection, data analysis, or interpretation of the data, or in the preparation, review, or approval of the manuscript for publication.

Conflict of interest All authors declare no support from any organisations for the submitted work. CG has received research funding from BMS Pfizer and Novo Nordisk, not related to this work. RJ reports he has received research funding, speaker honoraria, and consultancy fees from Astellas relating to enzalutamide and from Janssen relating to abiraterone. RJ has received consultancy fees from Janssen relating to other products not related to the work. RJ has received consultancy fees from Astellas relating to other products not related to the work. The other authors declare no competing interests.

Availability of data and material The CMOP management group are unable to share the raw data. Permission to view patient-level data needs to come from the UK Caldicott Guardian Council.

Code availability Available on request. Relevant permission, as mentioned for availability of data, needs to be obtained.

Author contributions All authors approve the final version. The authors contributed to this research study as follows: DR: conceptualisation, data curation, performed the analysis, interpretation, manuscript (writing, editing and reviewing); CG: conceptualisation, supervised the analysis, interpretation, manuscript (editing and reviewing); JP and TM: data linkage, interpretation, manuscript (reviewing); KB, JC, JL, $\mathrm{MB}, \mathrm{RJ}$, and $\mathrm{AB}$ : interpretation, manuscript (reviewing); OW: conceptualisation, supervised the analysis, interpretation, manuscript (editing and reviewing)

Ethics approval This was an observational study, so ethics approval was not required. However, the use of the data was approved by the Local Privacy Advisory Committee.

Consent to participate and publication Not applicable.

Open Access This article is licensed under a Creative Commons Attribution-NonCommercial 4.0 International License, which permits any non-commercial use, sharing, adaptation, distribution and reproduction in any medium or format, as long as you give appropriate credit to the original author(s) and the source, provide a link to the Creative Commons licence, and indicate if changes were made. The images or other third party material in this article are included in the article's Creative Commons licence, unless indicated otherwise in a credit line to the material. If material is not included in the article's Creative Commons licence and your intended use is not permitted by statutory regulation or exceeds the permitted use, you will need to obtain permission 
directly from the copyright holder. To view a copy of this licence, visit http://creativecommons.org/licenses/by-nc/4.0/.

\section{References}

1. International Agency for Research on Cancer-World Health Organisation. Cancer Today: Data visualization tools for exploring the global cancer burden in 2018 Global Cancer Observatory 2018. http://globocan.iarc.fr/. Accessed 30 June 2021.

2. Kirby M, Hirst C, Crawford E. Characterising the castrationresistant prostate cancer population: a systematic review. Int $\mathbf{J}$ Clin Pract. 2011;65(11):1180-92.

3. Scottish Medicines Consortium-Independent Review Panel. Abiraterone (Zytiga). SMC No. 764/12. 2012. https://www.scott ishmedicines.org.uk/media/1185/abiraterone_zytiga_resubmissi on_final_july_2012_amended_for_website.pdf. Accessed 30 June 2021.

4. Scottish Medicines Consortium- Independent Review Panel. Enzalutamide (Xtandi®). SMC No. 911/13. 2013. https://www. scottishmedicines.org.uk/media/1629/enzalutamide_xtandi_final_ october_2013 amended_081113.pdf. Accessed 30 June 2021.

5. Scottish Medicines Consortium- Independent Review Panel. Abiraterone (Zytiga $\left.{ }^{\circledR}\right)$. SMC No. 873/13. 2015. https://www.scott ishmedicines.org.uk/media/1186/abiraterone_zytiga_irp_final_ sept_2015_for_website.pdf. Accessed 30 June 2021.

6. Scottish Medicines Consortium- Independent Review Panel. Enzalutamide (Xtandi®). SMC No. 1066/15. 2016. https://www.scott ishmedicines.org.uk/media/1628/enzalutamide_xtandi_irp_final_ feb_2016_for_website.pdf. Accessed 30 June 2021.

7. Grochtdreis T, König H-H, Dobruschkin A, von Amsberg G, Dams J. Cost-effectiveness analyses and cost analyses in castration-resistant prostate cancer: a systematic review. PLoS ONE. 2018;13(12):e0208063.

8. Ellis LA, Lafeuille M-H, Gozalo L, Pilon D, Lefebvre P, McKenzie S. Treatment sequences and pharmacy costs of 2 new therapies for metastatic castration-resistant prostate cancer. Am Health Drug Benefits. 2015;8(4):185.

9. Koninckx M, Marco JL, Pérez I, Faus MT, Alcolea V, Gómez F. Effectiveness, safety and cost of abiraterone acetate in patients with metastatic castration-resistant prostate cancer: a real-world data analysis. Clin Transl Oncol. 2019;21(3):314-23.

10. Schultz NM, Flanders SC, Wilson S, Brown BA, Song Y, Yang $\mathrm{H}$, et al. Treatment duration, healthcare resource utilization, and costs among chemotherapy-naive patients with metastatic castration-resistant prostate cancer treated with enzalutamide or abiraterone acetate: a retrospective claims analysis. Adv Ther. 2018;35(10):1639-55

11. Ramaswamy K, Lechpammer S, Mardekian J, Huang A, Schultz NM, Sandin R, et al. Economic outcomes in patients with chemotherapy-naive metastatic castration-resistant prostate cancer treated with enzalutamide or abiraterone acetate plus prednisone. Adv Therapy. 2020;37:1-15.

12. Kreis K, Horenkamp-Sonntag D, Schneider U, Zeidler J, Glaeske G, Weissbach L. Treatment-related healthcare costs of metastatic castration-resistant prostate cancer in Germany: a claims data study. PharmacoEconomics Open. 2021;5(2):299-310.

13. The Scottish Government. Beating Cancer: Ambition and Action. 2016. https://www.gov.scot/binaries/content/documents/govsc ot/publications/strategy-plan/2016/03/beating-cancer-ambitionaction/documents/00496709-pdf/00496709-pdf/govscot\%3Adoc ument/00496709.pdf. Accessed 30 June 2021.

14. Baillie K, Mueller T, Pan J, Laskey J, Bennie M, Crearie C, et al. Use of record linkage to evaluate treatment outcomes and trial eligibility in a real-world metastatic prostate cancer population in Scotland. Pharmacoepidemiol Drug Saf. 2020;29(6):653-63.

15. CIS Oncology. ChemoCare goes live across NHS Scotland. 2012. https://www.cis-healthcare.com/latest/chemocare-goes-liveacross-nhs-scotland/. Accessed 30 June 2021.

16. Information Services Division- NHS National Services Scotland (NSS). SMR Datasets - SMR00 (Outpatient Attendance) 2019. https://www.ndc.scot.nhs.uk/Data-Dictionary/SMR-Datasets/ SMR00-Outpatient-Attendance/. Accessed 30 June 2021.

17. Information Services Division-NHS National Services Scotland (NSS). SMR Datasets-SMR01 (General/Acute Inpatient and Day Case). 2019.

18. National Records of Scotland. What We Do - About Us. 2019. https://www.nrscotland.gov.uk/about-us/what-we-do. Accessed 30 June 2021.

19. Information Services Division. Scottish Health Service Costs. 2018. https://www.isdscotland.org/health-topics/finance/ costs/. Accessed 30 June 2021.

20. Curtis LA. Burns, Amanda, Unit costs of health and social care 2018: personal social services research unit. Canterbury: University of Kent; 2018.

21. British National Formulary (online) [Internet]. BMJ Group and Pharmaceutical Press. 2018. www.medicinescomplete.com.

22. Geue C, Lewsey J, Lorgelly P, Govan L, Hart C, Briggs A. Spoilt for choice: implications of using alternative methods of costing hospital episode statistics. Health Econ. 2012;21(10):1201-16.

23. Charlson ME, Pompei P, Ales KL, MacKenzie CR. A new method of classifying prognostic comorbidity in longitudinal studies: development and validation. J Clin Epidemiol. 1987;40(5):373-83.

24. University of Manitoba. Concept: Charlson Comorbidity Index. 2019. http://mchp-appserv.cpe.umanitoba.ca/viewConcept.php? printer $=$ Y\&conceptID=1098. Accessed 30 June 2021.

25. Information Services Division. The Scottish Index of Multiple Deprivation (SIMD). 2019. https://www.isdscotland.org/produ cts-and-services/gpd-support/deprivation/simd/. Accessed 30 June 2021.

26. Mihaylova B, Briggs A, O'Hagan A, Thompson SG. Review of statistical methods for analysing healthcare resources and costs. Health Econ. 2011;20(8):897-916.

27. Glick HA, Doshi JA, Sonnad SS, Polsky D. Economic evaluation in clinical trials: OUP. Oxford: Oxford University Press; 2014.

28. Scottish Medicines Consortium. NHS Scotland Patient Access Scheme (PAS) Guidance. 2018. https://www.scottishmedicines. org.uk/making-a-submission/companies/patient-access-schem es/. Accessed 30 June 2021. 


\section{Authors and Affiliations}

Dikshyanta Rana ${ }^{1} \mathbb{D} \cdot$ Claudia Geue ${ }^{1} \cdot$ Kelly Baillie $^{2} \cdot$ Jiafeng Pan $^{3} \cdot$ Tanja Mueller $^{4} \cdot$ Jennifer Laskey $^{2}$. Marion Bennie $^{4,5}$. Julie Clarke ${ }^{2} \cdot$ Robert J. Jones $^{2,6} \cdot$ Ailsa Brown $^{7}$. Olivia Wu ${ }^{1}$

1 Health Economics and Health Technology Assessment (HEHTA), Institute of Health and Wellbeing, University of Glasgow, 1 Lilybank Gardens, Glasgow G12 8RZ, UK

2 Beatson West of Scotland Cancer Centre, NHS Greater Glasgow and Clyde, Glasgow, UK

3 Department of Mathematics and Statistics, University of Strathclyde, Glasgow, UK

4 Strathclyde Institute of Pharmacy and Biomedical Sciences, University of Strathclyde, Glasgow, UK
5 Clinical and Protecting Health Directorate, Public Health Scotland, Edinburgh, UK

6 Institute of Cancer Sciences, University of Glasgow, Glasgow, UK

7 Healthcare Improvement Scotland, Glasgow, UK 